\title{
Les Chironomidés (Diptera) du bassin de l'Oued Laou (Versant méditerranéen du Rif, Maroc)
}

\author{
K. Kettani ${ }^{1}$ \\ A. Vilchez-Quero 2 \\ D. Calle Martinez ${ }^{2}$ \\ T. El Ouazzani ${ }^{1}$
}

Mots clés : Diptera, Chironomidae, faunistique, biogéographie, Maroc.

Dans ce travail, on dresse un premier inventaire faunistique des Chironomidés du bassin de l'Oued Laou, rivière côtière méditerranéenne du versant nord-rifain du Maroc. 87 espèces ont pu être identifiées, dont 19 se révèlent nouvelles pour le Maroc.

The Chironomids (Diptera, Chironomidae) of the Oued Laou river (Mediterranean versant of the Rif, Morocco)

Keywords : Diptera, Chironomidae, faunistic, biogeography, Morocco.

In this work, the first faunistic inventory of the Chironomidae of Oued Laou is presented. The latter is a river of the north rifian versant of the mediterranean region of Morocco. 87 species have been identified, 19 are new records for Morocco.

\section{Introduction}

L'Oued Laou est l'une des plus importantes rivières de la côte méditerranéenne du Maroc ; il s'écoule dans la région Ouest du domaine rifain du SudOuest vers lë Nord-Est. Situé dans la région de Chefchaouen, il suit un cours sensiblement subméridien, dominé à l'Est par la dorsale calcaire et à l'Ouest par les hautes crètes du Rif.

Sa situation géographique présente une aire de contact intéressante entre plusieurs régions telles le sud de l'Europe et l'Afrique du Nord et présente ainsi une zone de passage entre les régions paléarctique et afrotropicale.

Les études consacrées aux Chironomidés du Maroc, avant 1986, sont récapitulées par Azzouzi \& Laville (1987) qui avaient dénombré 134 espèces.

Dans une étude plus récente effectuée dans ce dómaine (Azzouzi et al. 1992), de nouvelles récoltes de Chironomidés, essentiellement dans le réseau hydrographique de l'Oued Tensift (Marrakech),

1. Dept. de Biologie, Faculté des Sciences, Université Abdelmalek Essaadi, B.P. 2121 Tétouan, Maroc.

2. Dept. de Biologia Animal, Ecologia y Genética, Universidad de Granada, Granada, Espagne. ont porté à 223 le total des espèces actuellement recencées du Maroc.

Aucune étude suivie des Chironomidés n'a été jusqu'ici réalisée dans la région NordMéditerranéenne du Maroc. Ce travail représente donc le premier inventaire faunistique des Chironomidés de l'Oued Laou. Il s'inscrit dans le cadre d'une étude plus vaste concernant l'ensemble du Rif Marocain.

\section{Méthodes et stations étudiées}

Notre étude est basée exclusivement sur des exuvies nymphales recueillies à l'aide de filets à dérive de $250 \mu \mathrm{m}$ de vide de maille. Leur identification a été faite essentiellement à l'aide des clés de Lehmann (1972) pour les Eukiefferiella, Hirvenoja (1973) pour les Cricotopus, Wilson \& McGill (1982) pour les gen= res et surtout Langton (1991).

Le cours d'eau principal de l'Oued Laou est entièrement situé dans le Haut-Rif et constitue l'un des grands réseaux hydrographiques du versant Nordrifain. Les affluents les plus importants que reçoit ce bassin sont l'Oued Talembote, l'Oued Ouarra et l'Oued Maggo ; la longueur du Laou est de 154 km, le débit moyen annuel est de $13 \mathrm{~m}^{3} / \mathrm{s}$. et la superficie du bassin à l'embouchure est de $915 \mathrm{~km}^{2}$. 
Du point de vue géologique, le bassin de l'Oued Laou traverse les nappes de charriage de Chouamate, les chaînes calcaires, les nappes de charriage de Bni-Ider et l'unité de Tanger (Maurer 1968) et coupe dans un sol calcaire et marneux.

Les dépôts affleurant sont des limons du quaternaire avec des alluvions à granulométrie grossière.

Ce bassin est doté d'un climat méditerranéen à tendance humide, caractérisé par la succession de deux saisons très tranchées : un été chaud ct sec et une saison pluvieuse et fraîche qui va de la fin de l'automne au début du printemps. La pluviométrie moyenne annuelle est de $980 \mathrm{~mm}$ et la température moyenne annuelle est de $18^{\circ} \mathrm{C}$, ce qui traduit un climat doux et sec à basse altitude, frais et tcmpéré à haute altitude.

Le couvert végétal de ce bassin of fre une végétation arbustive, principalement représentee par Chamaerops humilis et Pistacia lentiscus. Les formations forestières sont représentées par Quercus suber, Quercus rotundifolia, Pinus pinaster et Cedrus atlantica.

Les récoltes des Chironomidés ont été faites dans 8 stations réparties sur le réseau hydrographique de l'Oued Laou (Fig. 1). Le choix de ces dernières a été fait en fonction des confluences principales, de l'altitude et de la pente ; ainsi les 8 stations prospectées s'échelonnent entre $425 \mathrm{~m}\left(\mathrm{~L}_{1}\right)$ et $35 \mathrm{~m}\left(\mathrm{~L}_{8}\right)$.
Les récoltes se sont échelonnées tous les deux mois : Octobre (O) et Décembre (D) 1991 ; Février (F), Avril (Av), Juin (J) et Août (A) 1992. Ainsi, chaque station a été prospectée 6 fois, soit un total de 48 échantillons étudiés.

Nous donnons les principales caractéristiques physico-chimiques et morphométriques de ces stations avec les valeurs moyennes des 6 campagnes de récoltes (Tableau 1) pour la température, le $\mathrm{pH}$, la conductivité, l'Oxygène, les Nitrates, le Calcium et les Phosphates.

Du fait des basses altitudes $(35-425 \mathrm{~m})$ des stations prospectées, la moyenne thermique est assez élevée ; son amplitude varie entre $11^{\circ} \mathrm{C}\left(\mathrm{L}_{1}\right.$ en Décembre) et $28^{\circ} \mathrm{C}\left(\mathrm{L}_{6}\right.$ en Août $)$, ce qui reflète des eaux relativement chaudes et légèrement alcalines, en ce qui concerne les valeurs de $\mathrm{pH}$.

Les valeurs moyennes de la conductivité sont basses et reflètent une pauvre minéralisation des eaux de ces stations.

Quant à l'oxygène, sa teneur moyenne pour chaque station, indique des eaux généralement propres. De même, les tencurs moyennes des nitrates et des phosphates, reflètent dans l'ensemble des eaux peu polluées.

Les teneurs en calcium sont légèrement élevées et traduisent le lessivage des terrains de la dorsale calcaire.

Tableau 1. Principales caractéristiques morphométriques et physico-chimiques des 8 stations de l'Oued Laou : P, pierres ou galets ; $S$, sable ; B, blocs ; G, graviers ; $r$, rapide ; $m$, modéré ; l, lent ; : moyennes des 6 campagnes de récolte.

Table 1. Principal morphometric and physico-chemical characteristics of 8 stations of the Oucd Laou : P, stoncs or pebbles ; S, sand ; $\mathrm{B}$, boulders ; G, gravels ; r, rapid ; $\mathrm{m}$, moderate ; $l$, slow ; "mean values of 6 field samples.

\begin{tabular}{|c|c|c|c|c|c|c|c|c|}
\hline & $L_{1}$ & $L_{2}$ & $L_{3}$ & $\mathrm{~L}_{4}$ & $\mathrm{~L}_{5}$ & $L_{6}$ & $L_{7}$ & $\mathrm{~L}_{8}$ \\
\hline Altitude (m) & 425 & 300 & 335 & 245 & 155 & 70 & 45 & 35 \\
\hline Pente (z) & 0,80 & 0.30 & 2.50 & 5 & 2,50 & 0.20 & 0.10 & 0.10 \\
\hline Nature ou courant & $m$ & $m$ & $m$ & $r$ & m & 1 & 1 & 1 \\
\hline Largeur (m) & 9 & 16 & 5 & 17 & 7 & 46 & 6 & 30 \\
\hline Substratum & $B / P$ & $G / S$ & B/P & B & $\mathrm{P} / \mathrm{G}$ & $5 / 6$ & $\rho$ & $S$ \\
\hline Temoérature $\left({ }^{\circ} \mathrm{C}\right)$. & 16.40 & 18.10 & 17.0 & 18.2 & 18.4 & 19.0 & 14.6 & 19.0 \\
\hline Conduclivilé (us/cm)" & 394 & 399 & $4 ! 7$ & 395 & 449 & 41 ! & 389 & 553 \\
\hline ohe & 8,02 & 8,08 & 7.72 & 8,01 & 8,02 & 8.10 & 8.24 & 7,95 \\
\hline $\mathrm{O}_{2}(\mathrm{mg} / \mathrm{l})=$ & 11,04 & 11.08 & 12.30 & 7.90 & 8.83 & 8.79 & 10.20 & 7.20 \\
\hline $\mathrm{NO}_{3}(\mathrm{mg} / 1)$ & 0,02 & 0.15 & 0.01 & 0.31 & 0.01 & 0.11 & 0.01 & 0.05 \\
\hline $\mathrm{Ca}^{++}(1 \mathrm{Omg} / 1) *$ & 13,96 & 13.15 & 14.20 & 12.73 & 13.72 & 12,80 & 15.40 & 14.24 \\
\hline $\mathrm{PO}_{4}{ }^{--}(\mathrm{mg} / \mathrm{l}) *$ & $0, \infty$ & 0.01 & 0.01 & 0.01 & 0.00 & 0.01 & 0.00 & 0.01 \\
\hline
\end{tabular}




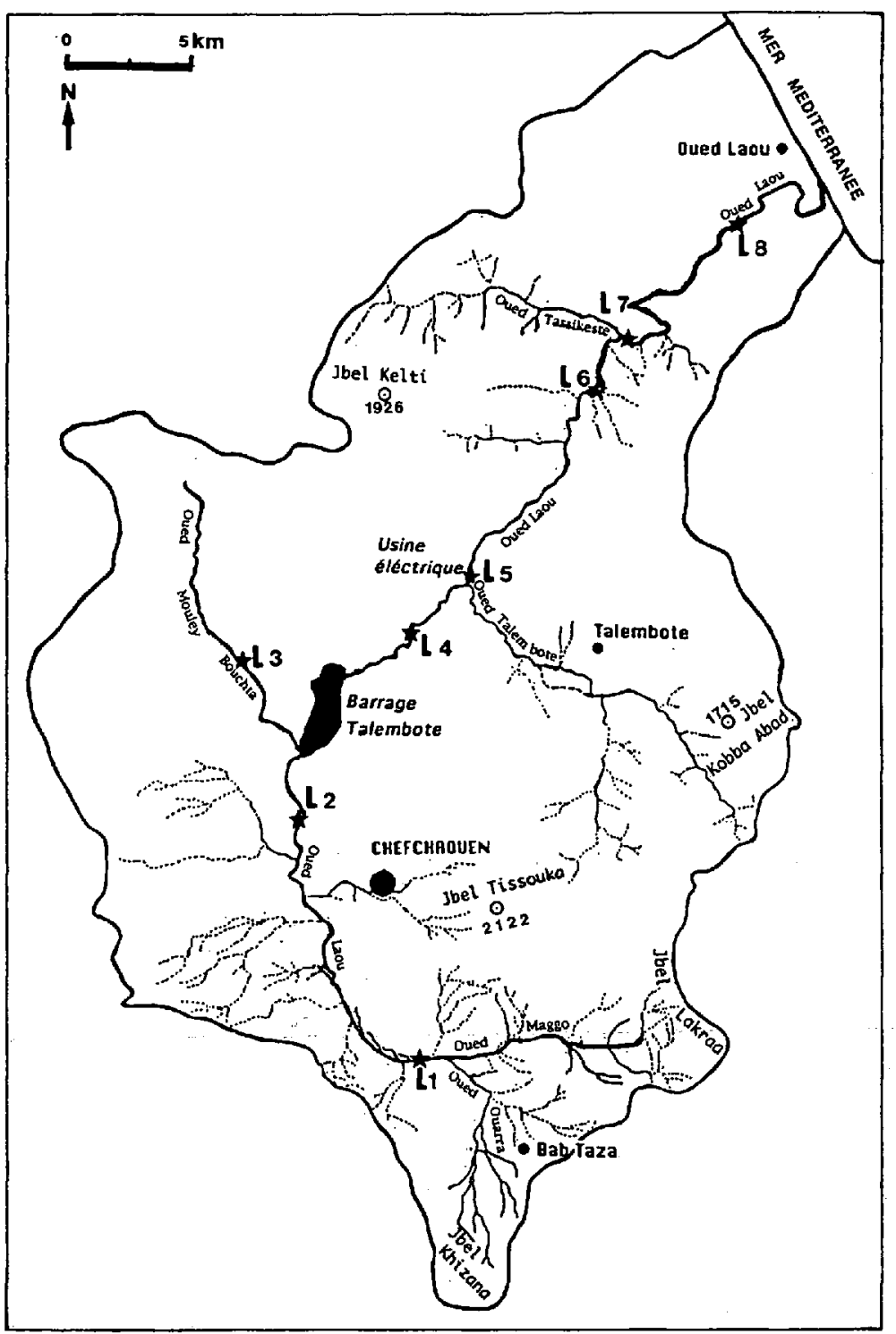

Fig. 1. Bassin de l'Oued Laou : localisation des 8 stations étudiées $\left(L_{1}\right.$ à $\left.L_{8}\right)$.

Fig. 1. Basin of the Oued Laou : location of the 8 stations $\left(\mathrm{L}_{1}\right.$ to $\left.\mathrm{L}_{8}\right)$.

\section{Résultats et discussion}

Au total, 87 espèces de Chironomidés ont été répertoriées au cours des 6 campagnes de récolte. Le tableau 2 récapitule leur liste avec leurs différentes stations et périodes de récoltes.
Ces 87 espèces se répartissent dans les principales sous-familles et tribus : Orthocladiinae $38 \%$, Chironomini $29 \%$, Tanytarsini $15 \%$, Tanypodinae $15 \%$, Diamesinae $2 \%$, Prodiamesinae $1 \%$. Les Chironominae et Orthocladiinae s'avèrent majoritaires par le nombre de leurs espèces par rapport aux autres sous-familles. 
Tableau 2. Liste et mois de récoltes des espèces de Chironomidés dans les 8 stations de l'Oued Laou.

* : Espèces nouvellement citée pour le Maroc.

Table 2. List and month of collection for species of chironomids of the Oued Laou, with number of species in parentheses. * : new species recorded for Morocco.

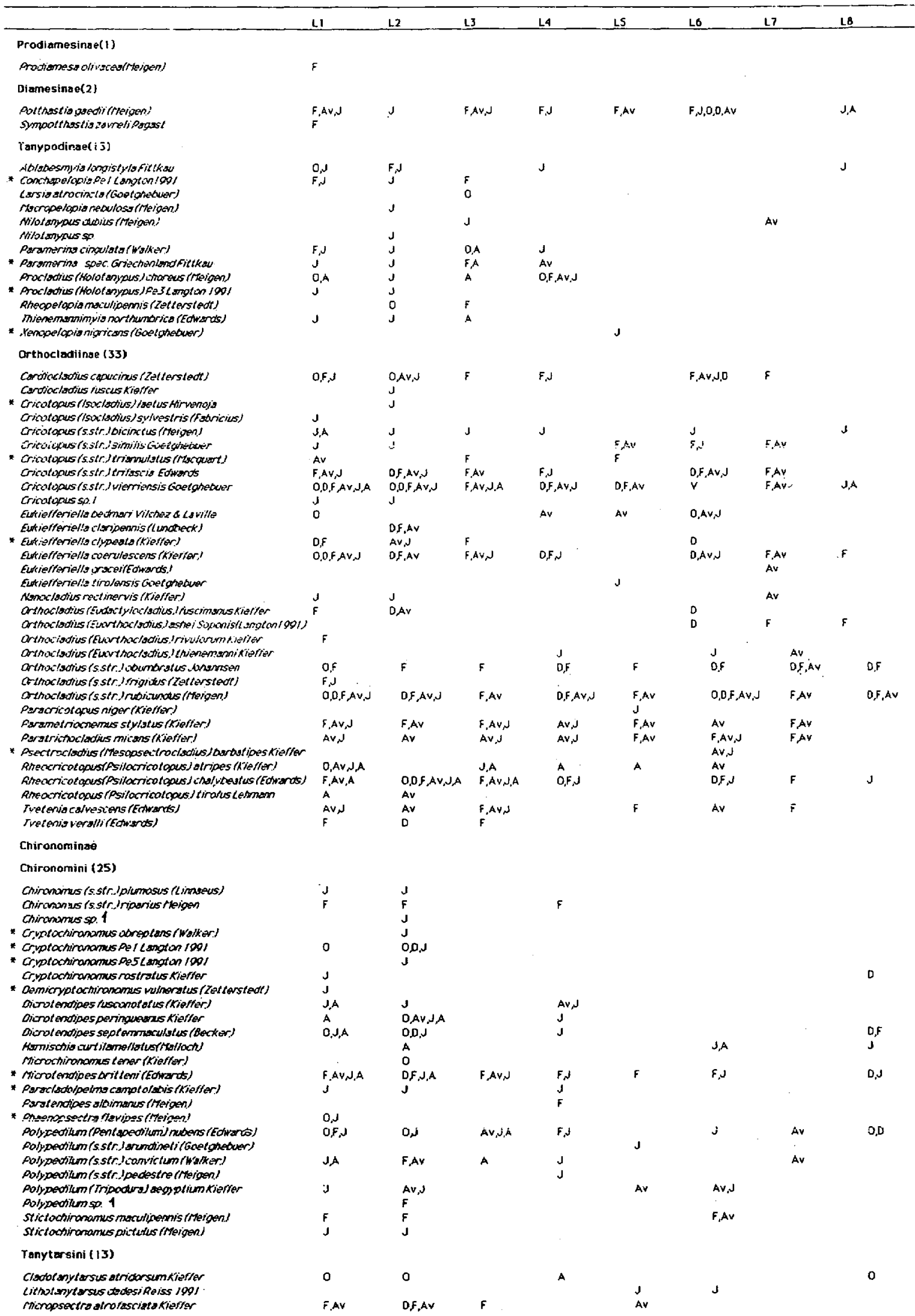


Tableau 2. Suite.

Table. 2. Next.

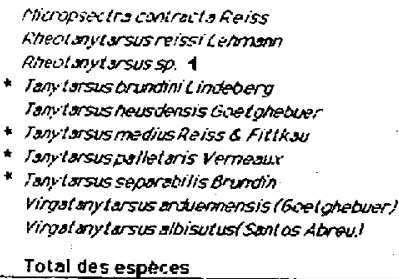

\begin{tabular}{|c|c|c|}
\hline$\left.O, D, F, A V_{1}\right]$ & $\begin{array}{l}O, D, F_{i} J, A \\
A\end{array}$ & $\begin{array}{l}\dot{F}, A \cup, J \\
A \\
A\end{array}$ \\
\hline$F, J$ & $J$ & $F, J$ \\
\hline $0^{\circ}$ & 0 & \\
\hline$J$ & $O, D, F, A Y, J, A$ & $F, A$ \\
\hline$s t$ & 58 & 33 \\
\hline
\end{tabular}

$0, A V, J$
$J$
$0, N$
$O, A$
$F, J$

J
D.F
J
A
F

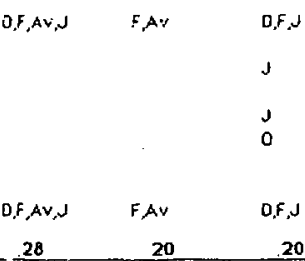

Parmi ces 87 espèces répertoriées dans l'Oued Laou, 18 sont nouvelles pour le Maroc et portent à un total de 241 les espèces connues à présent sur le territoire marocain depuis les derniers recensements bibliographiques (Azzouzi \& Laville 1987, Azzouzi et al. 1992).

Pour leur distribution géographique, la majorité a une répartition paléarctique $(88,5 \%)$, les autres ont une répartition méditerranéenne $(3,5 \%)$, afrotropicale $(4,5 \%)$, panpaléotropicale $(3,5 \%)$.

- Espèces méditerranéennes Eukiefferiella bedmari Paramerina spec. Griechenland Virgatanytarsus albisutus

- Espèces afrotropicales Dicrotendipes fusconotatus Dicrotendipes peringueyanus Paratrichocladius micans. Phaenopsectra flavipes

- Espèces panpaléotropicales Dicrotendipes septemmaculatus Microchironomus tener Polypedilum aegyptium

Certaines espèces dominantes du point de vue quantitatif, répartition spatiale et temporelle, appartiennent principalement à la sous-famille des Orthocladiinae : Orthocladius rubicundus $(30,4 \%)$, Orthocladius obumbratus $(15,6 \%)$, Cricotopus trifascia $(8,2 \%)$, Cardiocladius capucinus $(6,1 \%)$, Rheocricotopus chalybeatus $(4,1 . \%)$ et Cricotopus vierriensis $(2,8 \%)$. Orthocladius obumbratus ne fut présente que dans les récoltes de Décembre et de Février. Cricotopus trifascia dominait en Février et A ll les quatre autres furent récoltées durant toutes les périodes de l'année. D'autres espèces appartenant aux Chironominae telles Microtendipes britteni $(5,8 \%)$, Dicrotendipes peringueyanus (2 \%), Polypedilum nubens $(1,9 \%)$, Rheotanytarsus reissi

$(4,4 \%)$ et Virgatanytarsus albisutus $(2,9 \%)$, étaient présentes à toutes les périodes de récoltes avec une certaine prédominance pendant les mois les plus chauds.

Par ailleurs, la représentativité des autres espèces, par rapport aux précédentes, était relativement moindre. C'est le cas de Potthastia gaedii $(1,6 \%)$, Paramerina cingulata $(0,4 \%)$, Procladius choreus $(0,6 \%)$, Cricotopus bicinctus $(0,5 \%)$, Eukiefferiella coerulescens $(1,5 \%)$, Parametriocnemus stylatus $(0,4 \%)$, Polypedilum aegyptium $(0,3 \%)$ et Tanytarsus medius $(0,8 \%)$. Parmi ces dernières, certaines ne furent récoltées que durant quelques périodes déterminées : Parametriocnemus stylatus, Polypedilum aegyptium et Tanytarsus medius: Les autres étaient présentes à chaque période de récolte.

Lors des récoltes de Février, Avril et Juin en particulier, un maximum de richesse spécifique est enregistré (Tableau 3). Cette période coïncide avec la période dominante d'émergences de Chironomidés. Cette période aurait probablement deux pics, le premier en Février et le second en Juin. En effet, le nombre d'individus récoltés augmente considérablement pendant ces deux mois, alors qu'il baisse très netiement en Avril. Cette variation du nombre d'individus, semble liée aux cycles bivoltins ou trivoltins de certaines espèces. Un tel phénomène a été signalé récemment par Berg \& Hellenthal (1992).

Tableau 3. Nombre total des espèces de Chironomidés et nombre estimé d'individus recensés aux différents mois de récoltes.

Table 3. Specific abundance and number of individuals relative to the collecting months.

Nb. esp. : Total number of species

$\mathrm{Nb}$. Ind. : Estimated number of individuals.

\begin{tabular}{lcccccc}
\hline & Oct. & Déc. & Févr. & Avr. & Juin & Août \\
\hline Nb. espèces & 25 & 23 & 37 & 36 & 57 & 27 \\
Nb. individus & 600 & 8400 & 467000 & 2200 & 42400 & 660. \\
\hline
\end{tabular}


De point de vue richesse spécifique, les stations $\mathrm{L}_{1}$ et $\mathrm{L}_{2}$, les plus en amont, abritent durant toutes les périodes de récoltes un maximum d'espèces par rapport aux autres stations.

La très basse altitude qui caractérise les stations situées vers l'aval ( $\mathrm{L}_{7}$ et $\mathrm{L}_{8}$ ), semble à l'origine de la réduction de la richesse spécifique. En effet, on remarque que plus on monte en altitude, plus le nombre des espèces augmente.

Les stations $\mathrm{L}_{1}, \mathrm{~L}_{2}$ et $\mathrm{L}_{3}$ abritent certaines espèces non rencontrées dans les autres stations. Ainsi, la présence de Prodiamesa olivacea, Sympotthastia zavreli, Larsia atrocincta, Cricotopus sylvestris, Orthocladius rivulorum, Orthocladius frigidus, Demicryptochironomus vulneratus et Phaenopsectra flavipes se limite au niveau de $\mathrm{L}_{1}$ et celle de Macropelopia nebulosa, Rheopelopia maculipennis, Cricotopus laetus, Rheocricotopus tirolus, Chironomus plumosus, Cryptochironomus Pe 1 Langton 1991 et Stictochironomus pictulus au niveau de $\mathrm{L}_{2}$ et $\mathrm{L}_{3}$. Toutefois, ces espèces possèdent une large distribution altitudinale, de même que Eukiefferiella coerulescens, Tvetenia calvescens, Orthocladius rubicundus, Paratrichocladius stylatus et Micropsectra atrofasciata qui peuvent coloniser des zones altitudinales entre 400 et $2200 \mathrm{~m}$ : ce sont des espèces euryzonales et eurythermes (Laville \& Vinçon 1991).

Les différences dans la zonation altitudinale, observées ci-dessus, sur la répartition des espèces dans l'Oued Laou, pourraient être dues à la particularité du versant Nord-Est du croissant rifain caractérisé par un relief relativement élevé à faible distance de la mer : Jbel Kelti atteint $1926 \mathrm{~m}$ d'altitude à $18 \mathrm{~km}$, à vol d'oiseau, de la Méditerrannée. (Fig. 1).

En comparaison avec les espèces recensées dans le bilan faunistique de Azzouzi \& Laville 1987 et Azzouzi et al. 1992, $64 \%$ des espèces rencontrées dans l'Oued Laou sont communes avec celles citées par ces auteurs. $36 \%$ constituent non seulement des espèces nouvelles pour le Maroc, mais aussi pour l'Afrique du Nord, sauf pour 2 d'entre elles déjà signalées d'Algérie, Microtendipes britteni (Reiss 1977) et de Tunisie Phaenopsectra flavipes (Boumaïza \& Laville 1988).

Parmi les espèces signalées par Azzouzi et al. (1992) Lithotanytarsus dadesi Reiss 1991, également récoltée dans l'Oued Laou, est une forme très semblable à Lithotanytarsus emarginatus (Goetghebuer).
Elle est citée par Vilchez \& Lavandier (1986) et Calle Martinez (1994) au niveau du Guadalquivir et par Casas (1990) dans la Sierra-Nevada.

C'est une espèce endémique de la région sud-ouest paléarctique (Azzouzi et al. 1992).

La majorité des espèces nouvellement citées possèdent une répartition paléarctique. Paramerina spec. Griechenland a une répartition méditerranéenne et Dicrotendipes septemmaculatus une répartition panpaléotropicale. Cricotopus triannulatus et Paracladopelma camptolabis se distribuent aussi bien dans la région néarctique que paléarctique. Eukiefferiella clypeata, Microtendipes britteni, Phaenopsectra flavipes, Demicryptochironomus vulneratus, Tanytarsus brundini et Tanytarsus palletaris sont largement répandues dans toute la région paléarctique et, plus particulièrement, dans la région circum-méditerranéenne, alors que les cinq espèces ci-après ont une répartition paléarctique plus restreinte :

Xenopelopia nigricans: $\quad$ Espagne, France.

Psectrocladius barbatipes: France, Irlande.

Cryptochironomus obreptans :Italie, Angleterre.

Tanytarsus medius: $\quad$ France, Portugal.

Tanytarsus separabilis: $\quad$ Suède, Finlande.

Enfin, Conchapelopia Pe1, Procladius Pe 3 Langton 1991, Cricotopus laetus, Cryptochironomus Pe 1 Langton 1991 et Cryptochironomus Pe 5 Langton 1991 semblent intéressantes pour notre région d'étude car leur distribution est encore assez limitée. Procladius $\mathrm{Pe} 3$ a été signalée dans le Guadalquivir (Sierra de Cazorla, S-Espagne) par Calle Martinez (1994). Cricotopus laetus a été signalée en Finlande et au nord de la Russie.

Malgré la similitude altitudinale existante entre certaines stations des Oueds Laou et Tensift (Azzouzi et al. 1992), leurs peuplements chironomidiens se révèlent peu comparables. Parmi les 27 espèces recensées dans le Tensift, uniquement 7 ont été trouvées dans l'Oued Laou : Ablabesmyia longistyla, Nilotanypus dubius, Orthocladius thienemanni, Tvetenia calvescens, Dicrotendipes fusconotatus, Microchironomus tener et Virgatanytarsus arduennensis. Ce faible recoupement serait dû aux conditions écologiques différentes dans le HautAtlas et dans le Rif.

Par ailleurs, parmi les espèces Afrotropicales et Ethiopiennes mentionnées par Laville \& Reiss (1993), quatre espèces : Polypedilum aegyptium, 
Dicrotendipes peringueyanus, Dicrotendipes fusconotatus et Paratrichocladius micans ont été retrouvées dans l'Oued Laou. De même, parmi celles signalées par Cranston \& Armitage (1988) des Iles Canaries, seules deux espèces : Dicrotendipes septemmaculatus et Virgatanytarsus albisutus sont communes à ces deux régions.

La localisation géographique de l'Oued Laou ainsi que les particularités de sa faune chironomidienne mises en évidence dans ce travail incitent à envisager une étude faunistique comparative plus approfondie avec celle du Sud de l'Espagne. Une analyse comparative préliminaire montre par référence aux travaux de Laville \& Vilchez (1986), Vilchez \& Lavandier (1986), Casas (1990) et Calle Martinez (1994) que $74 \%$ de la faune recensée dans le Laou est connue des cours d'eau du Sud de l'Espagne.

Le tableau 4 récapitule les espèces de l'Oued Laou communes avec le reste du Maroc (Reiss 1977, Ram- dani \& Tourenq 1982, Azzouzi \& Laville 1987, Naya 1988, Azzouzi et al. 1992), la Cordillère bétique, (Calle Martinez 1994) et la région Afrotropicale. De même, les comparaisons entre les fréquences des sous-familles et tribus des Chironomidés des rivières de la péninsule ibérique les mieux étudiées et celles de l'Oued Laou (Tableau 5) révèlent une composition faunistique la plus ressemblante avec le Guadalquivir. Ceci peut être dû d'une part, à l'histoire géologique commune entre les deux régions et d'auitre part, à la similitude de leurs caractères écologiques et climatiques. Toutefois, l'analyse de ces résultats doit être considérée comme préliminaire.

\section{Travaux cités}

Azzouzi A. \& Laville H. 1987. - Premier inventaire faunistique des Chironomidés (Diptera, Chironomidae) du Maroc. Annis Limnol., 23 (3) : 217-224.

Azzouzi A., Laville H. \& Reiss F. 1992. - Nouvelles récoltes de Chironomidés (Diptera) du Maroc. Annls Limnol., 28 (3) : 225-232.

Tableau 4. Répartition des espèces de Chironomidés de l'Oued Laou et nombre des espèces communes avec d'autres régions.

* Comparaison avec la Cordillère Bétique (Calle Martinez 1994).

Table 4. Distribution of species of the Oued Laou and the number of common to other regions.

* Comparison with the results of Calle Martinez (1994) on the Cordillère Bétique.

\begin{tabular}{lcccc}
\hline & Oued Laou & $\begin{array}{c}\text { Reste du } \\
\text { Maroc }\end{array}$ & $\begin{array}{c}\text { Cordillère } \\
\text { Bétique }\end{array}$ & $\begin{array}{c}\text { Région } \\
\text { Afrotropicale }\end{array}$ \\
\hline Diamesinae & 2 & 2 & 2 & 0 \\
Tanypodinae & 13 & 8 & 7 & 0 \\
Orthocladiinae & 33 & 26 & 29 & 1 \\
Chironomini & 25 & 13 & 18 & 3 \\
Tanytarsini & 13 & 7 & 8 & 0 \\
\hline
\end{tabular}

Tableau 5. Pourcentage des especes des différentes sous-familles et tribus de Chironomidés rencontrés dans 3 réseaux d'Espagne (Sierra Nevada, Llobregat, Guadalquivir) et dans l'Oued Laou.

Table 5. Frequency of species of different taxa of chironomids collected in several rivers in Spain (Sierra Nevada, Llobregat, Guadalquivir) and the Oued Laou.

\begin{tabular}{|c|c|c|c|c|}
\hline & \multirow{3}{*}{$\begin{array}{c}\text { Tanypodinae } \\
\% \\
\end{array}$} & \multirow{3}{*}{$\begin{array}{l}\text { Orthocladiinae } \\
+ \text { Diamesinae } \\
\%\end{array}$} & \multicolumn{2}{|c|}{ Chironominae } \\
\hline & & & \multirow{2}{*}{$\begin{array}{c}\text { Tanyiarsini } \\
\% \\
\end{array}$} & \multirow{2}{*}{$\begin{array}{c}\text { Chironomirai } \\
\% \\
\end{array}$} \\
\hline & & & & \\
\hline Sierra Nevada & 8,5 & 66,0 & 14,4 & 11,1 \\
\hline Llobregat & 2,7 & 41,1 & 15,3 & 40,7 \\
\hline Guadalquivir & 9,1 & 53,3 & 12,1 & 24,8 \\
\hline oued Laou & 15,0 & 40,0 & 15,0 & 29,0 \\
\hline
\end{tabular}


Berg M.B. \& Hellenthal R.A. 1992. - Life histories and growth of lotic Chironomids (Diptera : Chironomidea). Ann. Entomol. Soc. Am., 85 (5) : 578-589.

Boumaïza M. \& Laville H. 1988. - Premier inventaire faunistique (Diptera, Chironomidae) des eaux courantes de la Tunisie. Annls Limnol., 24 (2) : 173-181.

Calle Martinez D. 1994. - Quironomidos (Diptera : Chironomidae) del alto Guadalquivir : estudio ecologico, faunistico y systematico. Ciclos nictemerales de emergencia. Tesis Doctoral, Univ. Granada, España : 366 p.

Casas J. 1990. - Estudio faunistico, ecologico y sistematico de los Quironomidos (Diptera, Chironomidae) de los rios de Sierra Nevada. Tesis Doctoral Univ. Granada, España :417 p.

Cranston P.S. \& Armitage P.D. 1988. - The Canary Islands Chironomidae described by T. Becker and by Santos Abreu. Dtsch. ent. Z., N.F., 35 (4-5) : 341-354.

Hirvenoja M. 1973. - Revision der Gattung Cricotopus van der wulp und ihrer Verwandten (Diptera, Chironomidae). Ann. Zool. Fennici, 10 : 1-363.

Langton P.H. 1991. - A key to pupal exuviae of WestPaléarctic Chironomidae. Cambridgeshire : 386 p.

Laville H. \& Vilchez A. 1986. - Les Chironomidés (Diptera) de quelques « lagunas » de haute altitude de la Sierra Nevada. (Granada, Espagne). Annis Limnol., 22 (1) : 53-63.

Laville H. \& Vinçon G. 1991. - A typological study of Pyrenean streams : Comparative analysis of the Chironomidae (Diptera) communities in the Ossau and Aure Valleys. Verh. Internat. Verein. Limnol., 24 : 1775-1784.
Laville H. \& Reiss F. 1993. - The Chironomid fauna of the mediterranean region reviewed. Netherlands journal of Aquatic Ecology, 26 (2-4) : 239-245.

Lehmann J. 1972. - Revision europaischer Arten (Puppen o und Imagines $\left.\sigma^{*}\right)$ der Gattung Eukiefferiella Thienemann. Beitr. Ent. Bd 22 : $718: 347-405$.

Maurer G. 1968. - Les Montagnes du Rif Central, Etude géomorphologique. Thèse de Doctorat Es Lettres. Paris, France : $481 \mathrm{p}$.

Naya A. 1988. - Peuplements chironomidiens (Diptera) du bassin du haut et moyen Sebou : Biotypologie et valeurs bioindicatrices. Thèse de 3ème cycle. Rabat, Maroc : $127 \mathrm{p}$.

Ramdani M. \& Tourenq J.N. 1982. - Contribution à l'étude faunistique de la Merja de Sidi Bou Ghaba. Chironomides (Diptères). Bull. Inst. scient. Rabat, $n^{\circ} 6: 179-185$.

Reiss F. 1977. - Verbreitungsmuster bei paläarktischen Chironomidenarten (Diptera, Chironomidae). Spixiana, $1: 85-97$.

Vilchez A. \& Lavandier P. 1986. - Composition et rythme journalier de la dérive des exuvies nymphales des Chironomidés dans le Guadalquivir (Sierra de Cazorla - Espagne). Annls Limnol., 22 (3) : 253-260.

Wilson R.S. \& McGill J.D. 1982. - A practical key to the genera of pupal exuviae of the British Chironomidae (Diptera, Insecta). University of Bristol: $62 \mathrm{p}$. 